INTERNATIONAL DESIGN CONFERENCE - DESIGN 2018

https://doi.org/10.21278/idc.2018.0192

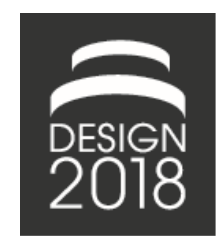

\title{
EXAMINING THE SOLUTION BIAS OF CONSTRUCTION KITS
}

\author{
J. Gopsill
}

\begin{abstract}
Prototyping is a fundamental activity across design with one of the most common types of tool used during prototyping sessions are construction kits. These kits have of a number of bricks that designers are able to construct designs from. Meeting the constraints within a kit ensures a valid solution. With the advent of high-performance computing, this paper computes the solution space that is represented by construction kits and investigates the number of pathways to potential solutions and reveals that construction kits have an inherent bias to particular solutions.
\end{abstract}

Keywords: prototyping, construction kits, combinatorics, constraint modelling, idea generation

\section{Introduction}

Prototyping is a fundamental design activity with studies showing that the method by which designers prototype along with the constraints set during prototyping sessions can affect design fixation and solution generation (Menold et al., 2017). Youmans (2011) highlights that prototyping can reduce the cognitive load on designers by constraining and providing an easily accessible method of interacting with the design space. Youmans (2011) also concluded that prototyping reduces design exploration and increases likelihood of design fixation. These findings have also been observed by Viswanathan and Linsey (2012) whose study revealed a potential correlation between the complexity of the design problem being represented by the prototyping tools and the level of exploration within the design space. Prototyping has also been used extensively in design processes where wider stakeholder engagement is desired. Coughlan et al. (2007) have shown the capability of prototyping to democratise the design process by providing a common platform to present the constraints of a design problem. This reduces the time spent communicating, discussing and defining the problem, and more time solving and generating potential solutions. To further democratise the process, Bennett et al. (2017) and Mathias et al. (2017) have investigated how to provide constraints in an increasingly immersive manner (Figure 1a). This has been through the embedding of constraints within the bricks of brick-style construction kits (e.g. $\mathrm{LEGO}^{\mathrm{TM}}$ ). By embedding the rules within the bricks, the environment is able to track and react in real-time with regards to the validity of the proposed solution.

Construction kits also enable individuals to explore and present designs to the same level of granularity and accuracy by providing ready-made constructs that can be combined together. This also removes the skill barrier one might have when using techniques, such as sketching, where the quality of the output can hinder the communication of the potential design (Craft and Cairns, 2009). Construction kits have been particularly successful in city, town, office and manufacturing facility configuration design tasks as well as developing problem solving skills during a child's development (Eisenberg et al., 2002; Hadhrawi and Larson, 2016). 


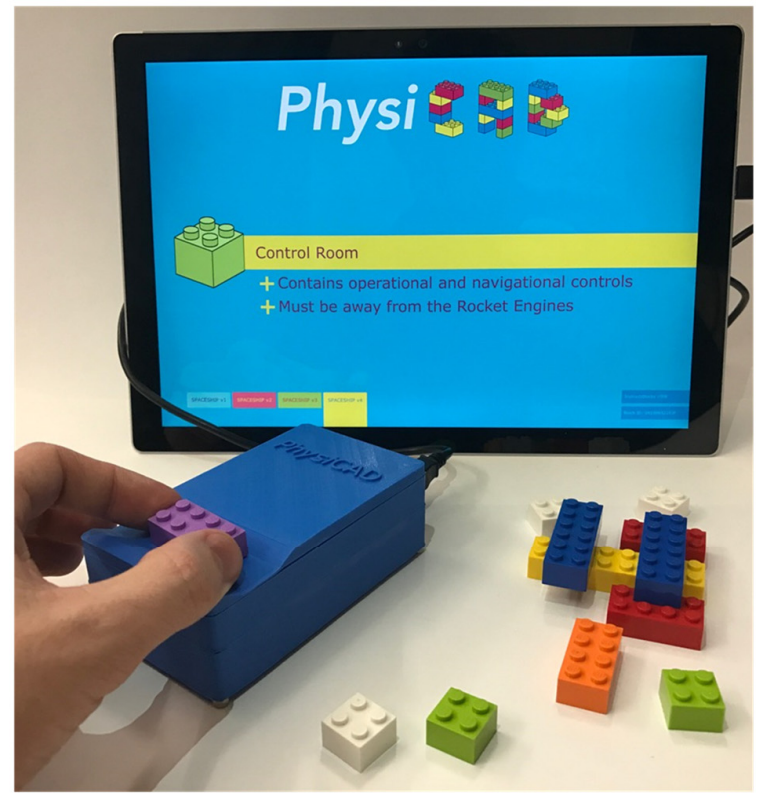

(a) Rules within bricks (Mathias et al., 2017)

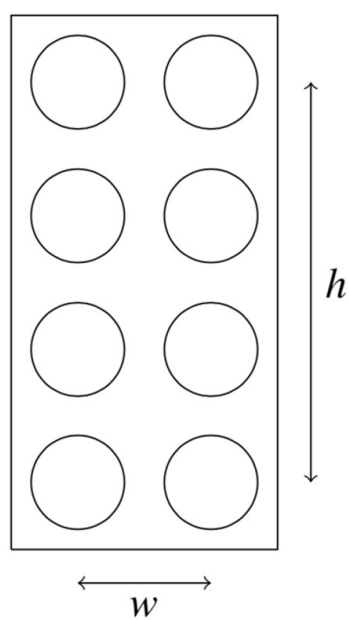

(b) Brick characteristics

Figure 1. Prototyping with construction kits

With descriptive studies revealing the profound effect prototyping tools have on the representation and exploration of the design space, further prescriptive studies into the development of the underlying theory of a construction kits suitability for design problems can now be performed. This can now be performed with the advent of high-performance computing, which enables the entire design space to be computed.

For example, mathematicians have examined the number of contiguous combinations of 2 wide by 4 high studded LEGO ${ }^{\text {TM }}$ bricks (Eilers, 2016) (Figure 1b). The analysis revealed that the original value of $103.0 \times 10^{6}$ contiguous combinations of six $2 \times 4$ bricks $\left(B_{(2,4)}(6)\right)$ as reported by LEGO ${ }^{\mathrm{TM}}$ was a factor of 9 out and that the true value is nearer $915.1 \times 10^{6}$. Although this informs us on the complete number of contiguous combinations of $B_{(2,4)}(6)$ bricks that can be created, it does not inform us on the number of pathways one could take to form these combinations and whether there are multiple pathways to achieve a particular combination. In addition, questions can also be asked as to whether construction kits contain an inherent combination bias with some combinations having a greater number of pathways than others. This may lead designers to favour particular solutions and not fully explore the design space. To investigate the number of pathways to combinations and answer the question of whether bias exists within current construction kits, this paper presents the results from a scoping study that has characterised the design space represented by $B_{(2,4)}(\mathrm{n})$ LEGO $^{\mathrm{TM}}$ bricks. This has been achieved by solving the combinatorial problem of constructing a breadth-first search algorithm that mimics the behaviour of constructing a combination from $B_{(2,4)}(\mathrm{n}) \mathrm{LEGO}^{\mathrm{TM}}$ bricks. By modelling the problem through the perspective of a designer, features such as the number of morphologically unique combinations and number of pathways to each combination can be derived. The analysis of the distribution of the pathways amongst the combinations can provide insights into the potential bias within the construction kit.

To continue, Section 2 defines and presents the breadth-first search algorithm that is used to solve the combinatorial problem of $B_{(2,4)}(\mathrm{n}) \mathrm{LEGO}^{\mathrm{TM}}$ bricks. This leads into Section 2, which defines the metrics used to determine the bias within construction kits. Section 3 presents the results from a study that has analysed the distribution of pathways across the potential combinations for $B_{(2,4)}(\mathrm{n})$. The role of constraints and their effects on any potential bias within the construction kit has been explored through 
the consideration of the addition of a No Rotation Constraint (NRC) case. Section 4 then discusses the potential bias in construction kits, how one can potentially control the bias, and future work this is developing a theory for the appropriate design and application of construction kits in design scenarios. The paper then concludes by highlighting the key findings from the scoping study.

\section{The combinatorics of $B_{(2,4)}(n)$ LEGO $^{\text {TM }}$ kits}

Before one can explore the potential bias within $B_{(2,4)}$ (n) LEGO ${ }^{\text {TM }}$ kits, it is important to be clear and precise in the definition of the combinatorics problem that is being solved. Therefore, this section defines the combinatorial problem as well as the approach that has been taken to solve the problem. The approach is a breadth-first search algorithm that has been designed to mimic the construction of a combination by a designer as well as enabling the inception of further constraints into the process of forming a combination.

\subsection{Defining the combinatorics problem}

A combination is to be made up of a set of bricks where a brick $(b)$ is defined by fixed dimensions w and $\mathrm{h}$ denoting the number of studs along the bricks width and height, respectively. All bricks are of the same depth $(z)$ and $w \leq h$. Therefore, a single brick can be denoted by $b_{(w, h)}$.

The bricks' top and bottom surfaces must be in parallel with the XY-plane with two sides parallel to the $\mathrm{x}$-axis (i.e. the bricks can only be in $90^{\circ}$ orientations). There is also the physical constraint of the bricks not being able to occupy the same space as one another. By meeting these constraints for $n$ bricks, a combination is formed. The set of combinations that meet all the constraints for a set of same type bricks is denoted as $B_{(w, h)}(n)$.

In solving the problem, there will be combinations that are rotationally symmetric. This has been explored by Eilers (2016). In this paper, the concept has been extended to identify morphologically equivalent combinations. I.e. combinations that are symmetric through reflection. It is argued that morphologically equivalent combinations are derivations of a single design. A feature that is often seen across Engineering Design with examples such as, left/right-handed versions of products and left/righthand drive vehicles.

Having defined the combinatorial problem, the paper continues by presenting the approach taken to solve the problem where consideration has been made to provide the ability to add rules to the construction kit.

\subsection{Solving the combinatorial problem}

The generalised process for solving the combinatorial of construction kits is described by Gopsill (2018). Therefore, this section describes the application of the process to solve for $B_{(w, h)}(n)$. LEGO ${ }^{\text {TM }}$ kits and is illustrated in Figure 2.

Step 1. defines the initial brick in space, which is defined as an object featuring $(x, y, z, w, h, \theta)$ where:

$x$ is the $\mathrm{x}$-coordinate of the leftmost line of studs;

$y$ is the $y$-coordinate of the uppermost line of studs;

$Z$ is the layer that the brick has been placed upon;

$w$ is the stud width;

$h$ is the stud height; and,

$\theta$ is the brick rotation.

Thus, the first brick in this problem is represented by $(0,0,0,2,4,0)$. 


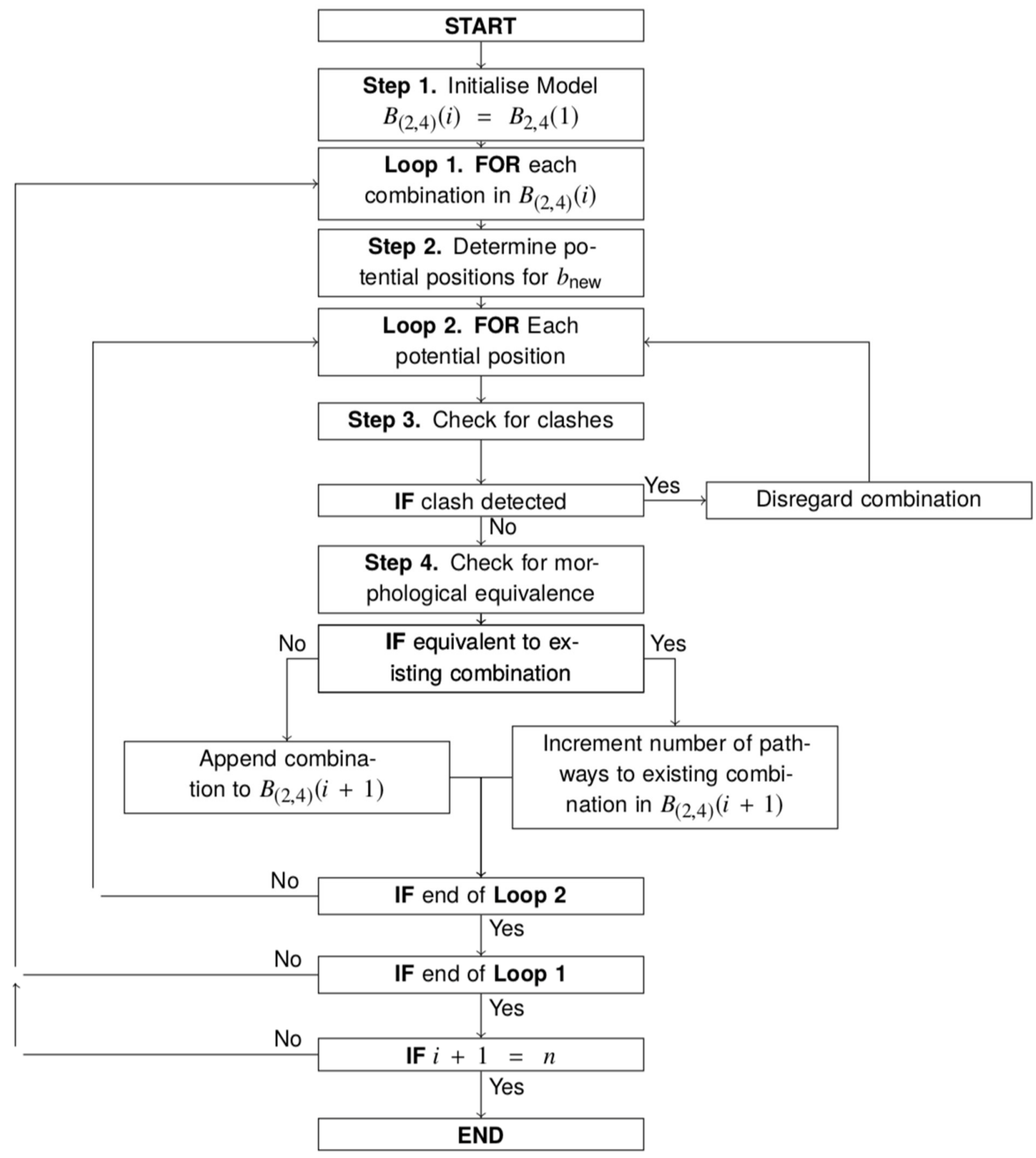

Figure 2. The process applied to solve the $B_{2,4}(n)$ combinatorial problem

With the first brick defined, the process can start to add bricks to the first combination. Running through each combination in the set of combinations $B_{(2,4)}(i)$ (Loop 1.) the process calculates all the possible positions that it could be placed by looking at all the bricks in the existing combination and creating an array of all the potential $x, y$ positions for $b_{\text {new }}$ (Step 2.). These positions are calculated using the ranges derived in Table 1.

Following the creation of an array of the potential positions for $b_{n e w}$, the process then checks whether the brick would intersect with any of the existing bricks in the combination. 
Table 1. Determining the potential brick positions for $\boldsymbol{b}_{\text {new }}$

\begin{tabular}{ccc}
\hline & $b_{\text {prev }}, \theta=0$ & $b_{\text {prev }}, \theta=90^{\circ}$ \\
\hline$b_{\text {new }}$ & $x_{\text {prev }}-w_{\text {new }}+1 \leq x_{\text {new }} \leq x_{\text {prev }}+w_{\text {prev }}$ & $x_{\text {prev }}-w_{\text {new }}+1 \leq x_{\text {new }} \leq x_{\text {prev }}+h_{\text {prev }}$ \\
$\theta=0$ & $y_{\text {prev }}-h_{\text {new }}+1 \leq y_{\text {new }} \leq y_{\text {prev }}+h_{\text {prev }}$ & $y_{\text {prev }}-h_{\text {new }}+1 \leq y_{\text {new }} \leq y_{\text {prev }}+w_{\text {prev }}$ \\
\hline$b_{\text {new }}$ & $x_{\text {prev }}-h_{\text {new }}+1 \leq x_{\text {new }} \leq x_{\text {prev }}+w_{\text {prev }}$ & $x_{\text {prev }}-h_{\text {new }}+1 \leq x_{\text {new }} \leq x_{\text {prev }}+h_{\text {prev }}$ \\
$\theta=90^{\circ}$ & $y_{\text {prev }}-w_{\text {new }}+1 \leq y_{\text {new }} \leq y_{\text {prev }}+h_{\text {prev }}$ & $y_{\text {prev }}-w_{\text {new }}+1 \leq y_{\text {new }} \leq y_{\text {prev }}+w_{\text {prev }}$ \\
\hline
\end{tabular}

\subsubsection{Detecting intersections (Step 3.)}

To detect whether any of the bricks intersect, the process uses a matrix representation for the layer that $b_{n e w}$ is being placed upon. The matrix is initialised as a zero array (Figure 3a) and the stud positions of the bricks that already exist on this layer as well as $b_{\text {new }}$ are added to the matrix. Once all the bricks have been added to the layer, intersects are identified if the matrix contains any elements that are $>1$ (Figure $3 b$ ). This indicates that the studs of multiple bricks are occupying the same space. If an intersection is detected then the combination is disregarded.
$\begin{array}{llllll}0 & 0 & 0 & 0 & 0 & 0\end{array}$
$\begin{array}{llllll}0 & 0 & 0 & 0 & 0 & 0\end{array}$
$\begin{array}{llllll}0 & 0 & 0 & 0 & 0 & 0\end{array}$
$\begin{array}{llllll}0 & 0 & 0 & 0 & 0 & 0\end{array}$
$\begin{array}{llllll}0 & 1 & 2 & 1 & 1 & 1\end{array}$
$\begin{array}{llllll}0 & 1 & 1 & 0 & 1 & 1\end{array}$
$\begin{array}{llllll}0 & 0 & 0 & 0 & 0 & 0\end{array}$
$\begin{array}{llllll}0 & 1 & 2 & 1 & 1 & 1\end{array}$
$\begin{array}{llllll}0 & 1 & 1 & 0 & 1 & 1\end{array}$
$\begin{array}{llllll}0 & 0 & 0 & 0 & 0 & 0\end{array}$
$\begin{array}{llllll}0 & 1 & 1 & 0 & 0 & 0\end{array}$
$\begin{array}{llllll}0 & 0 & 0 & 0 & 0 & 0\end{array}$
$\begin{array}{llllll}0 & 1 & 1 & 0 & 0 & 0\end{array}$
$\begin{array}{llllll}0 & 1 & 1 & 0 & 1 & 1\end{array}$
$\begin{array}{llllll}0 & 0 & 0 & 0 & 0 & 0\end{array}$
$\begin{array}{llllll}0 & 0 & 0 & 0 & 0 & 0\end{array}$
$\begin{array}{llllll}0 & 1 & 1 & 0 & 1 & 1\end{array}$
(a) Empty intersection matrix
(b) Intersection detected between two $b_{2,4}$
(c) No intersect detected between
$\begin{array}{llllll}0 & 0 & 0 & 0 & 0 & 0\end{array}$

Figure 3. Detecting clashes between bricks

This is performed for each position of $b_{\text {new }}$ and at the end of this loop, the process has generated all the possible combinations for $B_{(2,4)}(i+1)$.

\subsubsection{Detecting morphologically equivalent combinations (Step 4.)}

Having processed all the possible combinations of adding $b_{n e w}$, the process then identifies the number of morphologically unique combinations and in doing so, determines the number of pathways one can take to achieve a particular combination. This is achieved by calculating the following features for each combination:

- The sum pair-wise distances between the brick centres.

- The $\Delta$ in rotated to non-rotated bricks.

- The number of available studs.

For combinations to be morphologically equivalent, all these features have to equate. Figure 4 illustrates this for $B_{(2,4)}(2)$. where the 2 nd brick can only be added to the top of the 1st brick. Step 2. and 3. detects that there are 46 possible combinations for the bricks to be arranged. After checking for morphological equivalence, this is reduced to 14 morphologically unique combinations. 


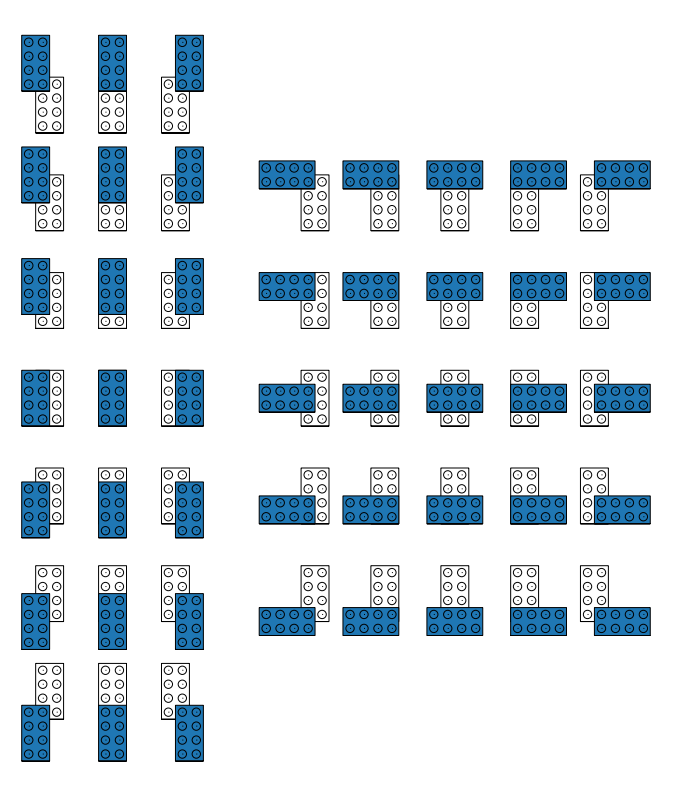

(a) 46 Combinations of $\boldsymbol{B}_{2,4}(\mathbf{2})$
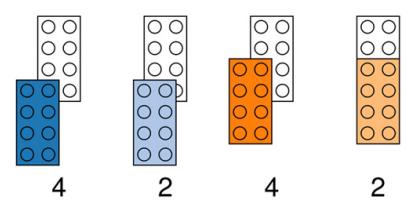

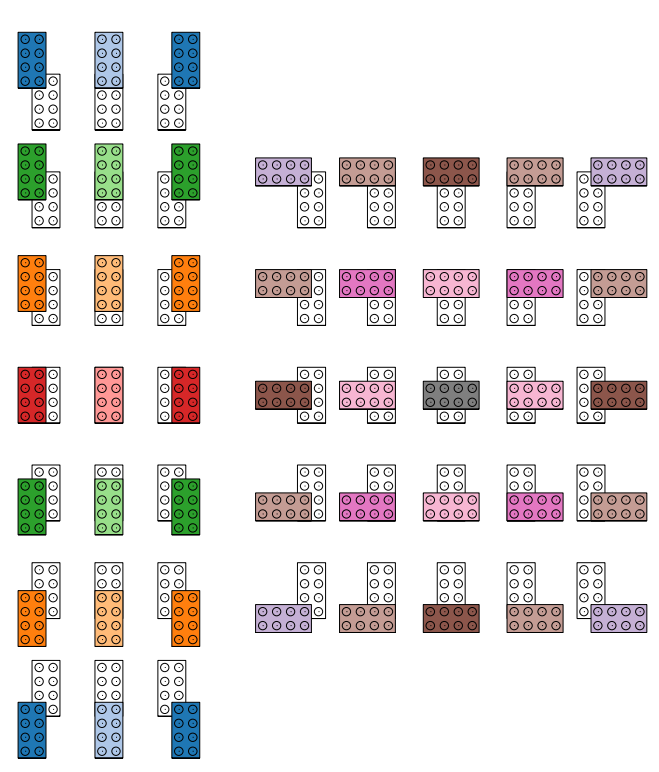

(b) 14 Morphologically unique combinations and their equivalent combinations of $B_{2,4}(2)$
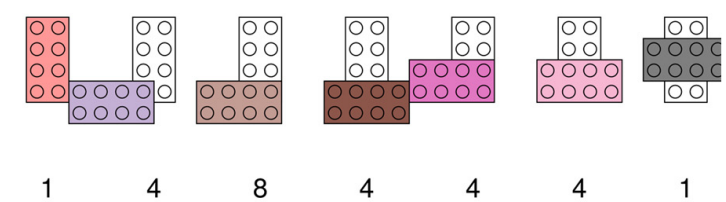

(c) Number of pathways to each combination

Figure 4. The combinations of $B_{2,4}(2)$

The process also provides the number of pathways one can take to form these combinations. This is illustrated in Figure $4 \mathrm{~b}$ where the bricks with the same colour have been detected as morphologically equivalent. Figure $4 \mathrm{c}$ shows that some combinations have 8 pathways, whilst others have only 1 . Hence, some of the combinations are statistically more likely to be generated by a designer and this is being driven by the nature of the construction kit. This initial result provides an indication that a bias to particular solutions is present in the construction kits designers use for prototyping and evidences the need for this study.

Once the morphologically unique combinations have been determined. The process repeats for the next brick and continues through this loop until the combinations for $B_{(2,4)}(n)$ have been generated.

\section{Examining the bias within $B_{(2,4)}(n)$ bricks}

To explore the potential bias, the paper uses the process described in Section 2.2 to solve the combinatorial problem for $B_{(2,4)}(n)$ bricks. From this, metrics on the distribution of the number of pathways amongst the morphologically unique combinations can be generated and will provide an indication of the potential bias within the construction kit. In addition, an investigation into the potential role additional constraints have on changing the bias within a construction kit is also examined. This has been achieved by preventing the rotation of the bricks during construction.

\subsection{Analysing the distribution of pathways to combinations}

To investigate the potential bias within a construction kit, an analysis of the distribution of the number of pathways $(P)$ to the number of morphologically unique combinations $(M)$ can be calculated. To 
achieve this, one can look at the Mean $\left(\frac{P}{M}\right)$ and the Gini coefficient $(G)$ that can be derived from the Lorenz curve of the distribution of pathways amongst the combinations of $B_{(2,4)}(n)$ (Figure 5).

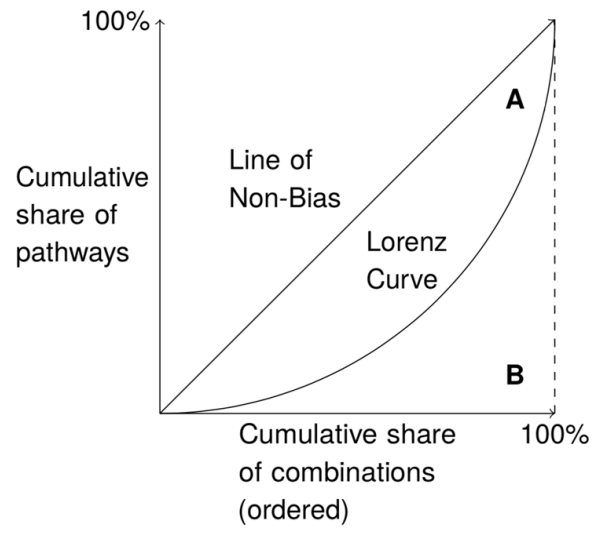

Figure 5. Lorenz curve of the distribution of pathways to combinations

Where the Gini coefficient is given by:

$$
G=\frac{\sum_{i=1}^{n} \sum_{j=1}^{n}\left|x_{i}-x_{j}\right|}{2 \sum_{i=1}^{n} \sum_{j=1}^{n} x_{j}}
$$

Where $x_{i}$ is the wealth of person $i, x_{j}$ is the wealth of person $j$ and $n$ is the number of people. In terms of the Lorenz curve in Figure 5, this is the ratio of the area under the Lorenz curve (B) and the area for a non-biased kit $(\mathbf{A}+\mathbf{B})$.

A Gini coefficient of 0 indicates that the number of pathways is equally distributed across the morphologically unique combination. This would indicate that no bias exists within the construction kit. In contrast, a highly biased construction kit is where one combination has all the pathways.

For the case of analysing construction kits, additional adaptations to the Gini coefficient have to be made due to the following features:

- One Pathway Minimum: All combinations must have at least one pathway otherwise the combination would not exist in the population.

- Discrete Allocation Pathways: cannot be split beyond an integer level. Therefore, the remainder of the modulus has to be split as evenly as possible.

Taking these constraints into account yields the following results for the two extreme cases of an entirely biased and non-biased construction kit would yield areas for a biased and un-biased kit, respectively:

$$
\begin{aligned}
& A_{\text {biased }}=\frac{q M^{2}+r}{2} \\
& A_{\text {non-biased }}=\frac{M^{2}+(P-M)}{2}
\end{aligned}
$$

Where $M$ is the number of morphologically unique combinations, $P$ is the number of pathways to the combinations, and $q \& r$ are the quotient and remainder of $P / M$ respectively.

These values can then be used to normalise against the area $(\mathbf{B})$ calculated for $B_{(2,4)}(n)$ to form the Gini coefficient.

$$
\mathrm{G}=1-\frac{\boldsymbol{B}-A_{\text {biased }}}{A_{\text {biased }}-A_{\text {non-biased }}}
$$

\section{2. $B_{(2,4)}(n)$ bricks}

To analyse the bias within $B_{(2,4)}(n)$, the process was deployed on the High-Performance Computing (HPC) facility at the University of Bath. The system contains 3,072 cores, 18TB main memory and $300 \mathrm{~TB}$ storage, and has a peak performance of 57Tflops. The maximum job size reported in this paper was of 64 cores running for a period of 6 hours. 
Table 2 presents the results for both the no additional constraint (NAC) and no rotation constraint (NRC) scenarios. Focusing on the NAC scenario, it can be seen that $\mathrm{n}$ has a large factor in the number of morphologically unique combinations and pathways one can create and use. Although this is a sensible conclusion as you're increasing the degrees of freedom within the construction kit, the rate at which it increases appears to be at an exponential rate. Looking at the mean, it can be seen that this is also increasing, which reveals that the number of pathways is increasing at a higher rate than the number of combinations. Taking the perspective of a designer, this would give the illusion that the number of potential solutions is much greater than there actually is. Looking at $P_{\min }$ and $P_{\max }$, which are the minimum and maximum number of pathways to a combination within the set, it can be seen that $P_{\min }$ remains fairly consistent and low with some combinations having very few pathways to them. In comparison, $P_{\max }$ increases at a similar rate to the number of pathways and combinations, and shows that there are some combinations that are much more likely to be attained by the designer. This is further confirmed by $G$, which indicates there is indeed a bias within the LEGO ${ }^{\mathrm{TM}}$ construction kit and as $\mathrm{n}$ increases, $G$ increases revealing that the bias builds within the kit.

Table 2. Number of pathways and morphologically equivalent combinations of $B_{2,4}(n)$

\begin{tabular}{|c|c|c|c|c|c|c|c|c|c|}
\hline$B_{w, h}(n)$ & $B$ & $M$ & $\frac{B}{M}$ & $P_{\text {min }}$ & $P_{\max }$ & $A_{\text {non-biased }}$ & $A_{\text {biased }}$ & B & $G$ \\
\hline \multicolumn{10}{|l|}{ NAC } \\
\hline$B_{24}(2)$ & 92 & 14 & 6.6 & 2 & 16 & 620 & 137 & 472 & 0.31 \\
\hline$B_{2,4}(3)$ & $12.3 \times 10^{3}$ & 429 & 28.7 & 4 & 104 & $2.6 \times 10^{6}$ & $98.0 \times 10^{3}$ & $1.6 \times 10^{6}$ & 0.39 \\
\hline$B_{2,4}(4)$ & $2.2 \times 10^{6}$ & $33.3 \times 10^{3}$ & 66.6 & 4 & 832 & $36.9 \times 10^{9}$ & $557.2 \times 10^{6}$ & $21.4 \times 10^{9}$ & 0.42 \\
\hline$B_{2,4}(5)$ & $497.8 \times 10^{6}$ & $3.0 \times 10^{6}$ & 166.2 & 4 & $14.9 \times 10^{3}$ & $744.8 \times 10^{12}$ & $4.5 \times 10^{12}$ & $370.8 \times 10^{12}$ & 0.51 \\
\hline \multicolumn{10}{|l|}{ NRC } \\
\hline$B_{2,4}(2)$ & 42 & 8 & 5.3 & 2 & 8 & 162.0 & 49.0 & 131.0 & 0.27 \\
\hline$B_{2,4}(3)$ & $2.5 \times 10^{3}$ & 139 & 17.9 & 4 & 32 & $172.5 \times 10^{3}$ & $10.8 \times 10^{3}$ & $126.0 \times 10^{3}$ & 0.29 \\
\hline$B_{24}(4)$ & $198.5 \times 10^{3}$ & $4.6 \times 10^{3}$ & 43.5 & 4 & 448 & $449.7 \times 10^{6}$ & $10.5 \times 10^{6}$ & $300.6 \times 10^{6}$ & 0.34 \\
\hline$B_{2,4}(5)$ & $19.7 \times 10^{6}$ & $193.4 \times 10^{3}$ & 101.6 & 4 & $2.0 \times 10^{3}$ & $1.9 \times 10^{12}$ & $18.7 \times 10^{9}$ & $1.1 \times 10^{12}$ & 0.42 \\
\hline
\end{tabular}

The addition of the no rotation constraint reveals a reduction in the number of pathways and morphological unique combinations for equivalent $\mathrm{n}$ bricks. However, the rate of increase does climb considerably and follows a similar trajectory to the NAC case (Figure 6a). Looking at the Gini coefficient, the addition of the fixed rotation constraint does increase the equality of the system for equivalent $n$ bricks but the increase in bias as $n$ increases is still observed. Figure $6 \mathrm{~b}$ highlights that the addition of the no rotation constraint provides a translation to the Gini coefficient line.

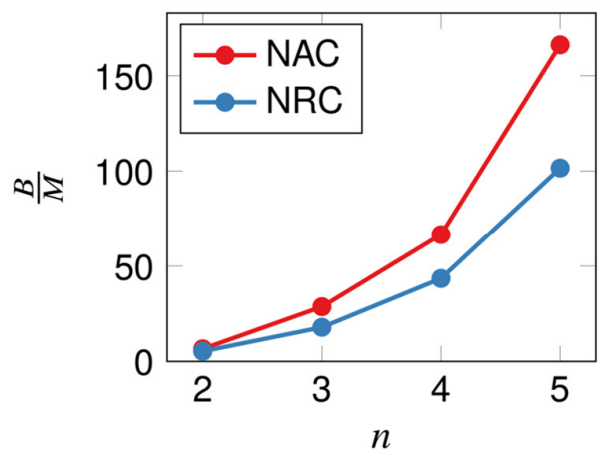

(a) Mean pathway to combination ratio

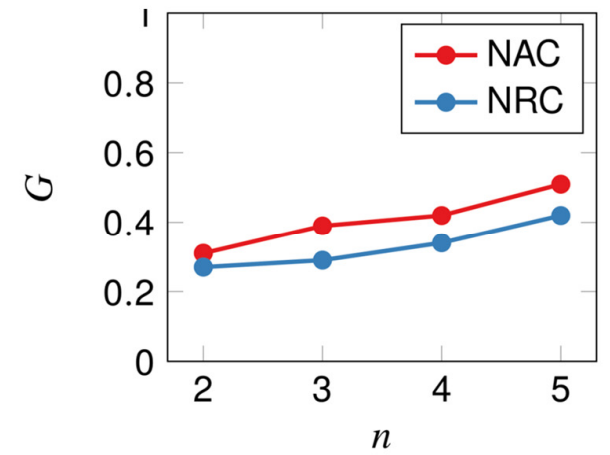

(b) Gini Coefficient

Figure 6. Characteristics of bias within $B_{2,4}(n)$ construction kits 


\section{Discussion and future work}

These initial results do reveal that biases exist in the LEGOTM construction kit that has been used in many design scenarios around the world (Brereton and McGarry, 2000; Ringwood et al., 2005; Bethke Wendell and Rogers, 2013). It has been observed that the bias increases as the number of bricks increases. It is also interesting to see the exponential rate at which the design space increases as bricks are added to the combination. Further, the discovery of the number of pathways increases at a higher rate than the number of combinations suggests that there may be a perception of more potential solutions than actual solutions. This 'perceived design freedom' within a construction kit may have an impact on the cognitive loading on a designer and their ability to conceptualise potential designs before they realise them with the kit.

This poses further questions with respect to the role that construction kits have had on the design of products and what would have been if kits featured different biases. For example, does bias:

- effect design fixation?

- change how much of the design space is explored?

- lead to different solutions?

And, how much of an effect has popular construction kits had on shaping designs across society and the way society construct and solve problems? These results show that further research is required in the analysis of construction kits to fully answer these questions.

In addition, there are the human factors involved in using these construction kits. This is an area that the author is actively working upon. It would be interesting to see whether the distribution of the final designs from designers and/or individuals across society correlates with the statistical distribution generated by the combinatorial algorithm. An initial hypothesis is that individuals may be more inclined to use two or more studs when constraining a brick as one stud provides rotational freedom to a brick. One can also go further to analyse aspects of co-creation around construction kits and begin to unravel how it supports and/or hinders design space exploration.

To further support these studies, research is required on the metrology of construction kits in order to determine a bricks position with respect to the other bricks in the combination. Achieving this would enable researchers to capture the sequence of operations a designer performs to construct a design. This information could then be directly correlated with the outputs from the combinatorial algorithm. Further, the combinatorial algorithm needs to be developed further to account for combinations that may be physically possible but would be impossible for an individual to build due to the occlusion of the existing bricks in the structure. Through this, a theory could be developed, which would enable the design of construction kits that can further support future design activities.

\section{Conclusion}

Prototyping is a fundamental activity across design with many descriptive studies investigating its ability to support design. One of the most common types of tool used during prototyping sessions are construction kits, such as LEGO ${ }^{\mathrm{TM}}$. These kits have a number of bricks that designers are able to construct designs from. As long as the constraints are met, a valid design has been achieved. Descriptive studies have concluded that construction kits have been instrumental in enabling wider stakeholder engagement and enable designers to focus on particular elements of the design problem.

However, it is posited that how the problem is represented by the construction kit has the potential to introduce an unknown bias where there is statistically more chance for certain solutions to be generated than others. This has been proven by this paper through its scoping study into the construction combinations of $\mathrm{n}, 2$ wide, 4 high LEGO $^{\mathrm{TM}}$ bricks. Through solving the combinatorial problem, the paper has revealed that biases do exist within $B_{(2,4)}(n)$ construction kits. The addition of constraints reduces the design space and decreases the bias for the same n, however the increase in bias still remains as $n$ increases. Even-though a bias is present, this may be hidden from a designer due to the exponential growth rate of potential combinations as one adds more bricks to the system. Thus, further studies are required on the human factors involved in constructing a combination using a kit to evaluate this.

These findings also pose further questions into the bias of other construction kits and how one might want to control bias within a construction kit for given design scenarios. In addition, as these kits are 
used by infants to develop problem solving skills, these results reveal that society has been continually designing with systems that contain bias towards particular solutions. If one were to build a new construction kit that altered this bias, what societal changes and/or effects would it have on the development of problem solving cognitive abilities? These questions are now able to be explored with computing power made available through HPC facilities.

\section{Acknowledgements}

This research made use of the Balena High Performance Computing (HPC) Service at the University of Bath.

\section{References}

Bennett, P., Fraser, M., Boa, D. and Hicks, B. (2017), "InstructiBlocks: Designing with Ambiguous PhysicalDigital Models", Proceedings of the Eleventh International Conference on Tangible, Embedded, and Embodied Interaction - TEI '17, Yokohama, Japan, March 20-23, 2017, ACM, New York, pp. 551-558. https://doi.org/10.1145/3024969.3025092

Bethke Wendell, K. and Rogers, C. (2013), "Engineering design-based science, science content performance, and science attitudes in elementary school", Journal of Engineering Education, Vol. 102 No. 4, pp. 513-540. https://doi.org/10.1002/jee.20026

Brereton, M. and McGarry, B. (2000), “An observational study of how objects support engineering design thinking and communication: implications for the design of tangible media", Proceedings of the SIGCHI conference on Human Factors in Computing Systems- CHI '00, The Hague, The Netherlands, April 1-6, 2000, ACM, New York, pp. 217-224. https://doi.org/10.1145/332040.332434

Coughlan, P., Suri, J.F. and Canales, K. (2007), "Prototypes as (Design) Tools for Behavioral and Organizational Change: A Design-Based Approach to Help Organizations Change Work Behaviors”, The Journal of Applied Behavioral Science, Vol. 43 No. 1, pp. 122-134. https://doi.org/10.1177/0021886306297722

Craft, B. and Cairns, P. (2009), "Sketching: Outlines of a Collaborative Design Method", Proceedings of the 23rd British HCI Group Annual Conference on People and Computers: Celebrating People and Technology BCSHCI '09, Cambridge, UK, September 1-5, 2009, British Computer Society, Swindon, UK, pp. 65-72.

Eilers, S. (2016), “The LEGO Counting Problem”, The American Mathematical Monthly, Vol. 123 No. 5, pp. 415426. https://doi.org/10.4169/amer.math.monthly.123.5.415

Eisenberg, M., Eisenberg, A., Gross, M., Kaowthumrong, K., Lee, N. and Lovett, W. (2002), "Computationallyenhanced construction kits for children: Prototype and principles", Proceedings of the Fifth International Conference of the Learning Sciences - ICLS 2002, Seattle, USA, October 23-26, 2002, pp. 79-85.

Gopsill, J.A. (2018), "Design Freedom of Construction Kits through Morphological Equivalence, Perceived Freedom and Combination Equality", Royal Society Open Science, (submitted).

Hadhrawi, M. and Larson, K. (2016), "Illuminating LEGOs with Digital Information to Create Urban Data Observatory and Intervention Simulator", Proceedings of the 2016 ACM Conference Companion Publication on Designing Interactive Systems - DIS '16 Companion, Brisbane, QLD, Australia, June 4-8, 2016, ACM, New York, pp. 105-108. https://doi.org/10.1145/2908805.2909400

Mathias, D., Boa, D., Hicks, B., Snider, C., Bennett, P. and Taylor, C. (2017), "Design variation through richness of rules embedded in LEGO bricks", Proceedings of the 21st International Conference on Engineering Design -ICED '17, Vol 8: Human Behaviour in Design, Vancouver, Canada, August 21-25, 2017, The Design Society, Glasgow, pp. 99-108.

Menold, J., Jablokow, K. and Simpson, T. (2017), "Prototype for X (PFX): A holistic framework for structuring prototyping methods to support engineering design", Design Studies, Vol. 50, pp. 70-112. https://doi.org/10.1016/j.destud.2017.03.001

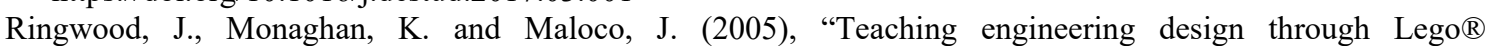
MindstormsTM", European Journal of Engineering Education, Vol. 30 No. 1, pp. 91-104. https://doi.org/10.1080/03043790310001658587

Viswanathan, V.K. and Linsey, J.S. (2012), "Physical Models and Design Thinking: A Study of Functionality, Novelty and Variety of Ideas", Journal of Mechanical Design, Vol. 134 No. 9, pp. 091004. https://doi.org/10.1115/1.4007148

Youmans, R.J. (2011), "The effects of physical prototyping and group work on the reduction of design fixation", Design Studies, Vol. 32 No. 2, pp. 115-138. https://doi.org/10.1016/j.destud.2010.08.001

Dr. James Gopsill, Lecturer in Engineering Design

University of Bath, Mechanical Engineering

Claverton Down, BA2 7AY Bath, United Kingdom

Email: J.A.Gopsill@bath.ac.uk 